PROCEEDINGS OF THE AMERICAN MATHEMATICAL SOCIETY

Volume 126, Number 3, March 1998, Pages 899-905

S 0002-9939(98)04185-9

\title{
ENVELOPING SEMIGROUPS AND MAPPINGS ONTO THE TWO-SHIFT
}

\author{
KENNETH BERG, DAVID GOVE, AND KAMEL HADDAD \\ (Communicated by Mary Rees)
}

\begin{abstract}
Enveloping semigroups of topological actions of semigroups $G$ on compact spaces are studied. For zero dimensional spaces, and under modest conditions on $G$, the enveloping semigroup is shown to be the Stone-Čech compactification if and only if some Cartesian product has the two-shift as a factor. Examples are discussed showing that, unlike in the measure theory case, positive entropy does not imply the existence of such a factor even if the Cartesian product has large entropy.
\end{abstract}

Let $G$ be a discrete multiplicative semigroup with at least one element $g_{0}$ such that $g \rightarrow g_{0} g$ is injective. Let $X$ be a compact Hausdorff space. We say $\sigma: G \times X \rightarrow$ $X$, written $(g, x) \rightarrow \sigma_{g}(x)$, is an action of $G$ on $X$, and we say $(X, G, \sigma)$ is a $G$-flow, if $\sigma_{g h}=\sigma_{g} \circ \sigma_{h}$ for all $g, h \in G$. We sometimes refer to the flow $X$ when $G$ and $\sigma$ are understood. We will assume that if $g_{1} \neq g_{2}$ then there is at least one $x \in X$ such that $\sigma_{g_{1}}(x) \neq \sigma_{g_{2}}(x)$. Given a $G$-flow $X$ let $E=E_{X}$ be the closure of $\left\{\sigma_{g}: g \in G\right\}$ in $X^{X}$ with the product topology. Clearly $E$ is compact and it can easily be shown that $E$ is a subsemigroup of $X^{X}$ where $(\xi \eta)(x)=\xi(\eta(x))$ for $\xi, \eta \in X^{X}$ and $x \in X$. $E$ is called the enveloping semigroup of $X$ (the enveloping semigroup was first introduced by Ellis in [3]. Other sources in the literature dealing with enveloping semigroups include [1], [4] and [5]). Let $G_{E}=\left\{\sigma_{g}: g \in G\right\}$. Observe $g \rightarrow \sigma_{g}$ is an injection of $G$ onto the dense subset $G_{E}$ of $E$.

Let $C$ be the set of all continuous real valued functions with domain $X$ and let $B$ be the set of all bounded real valued functions with domain $G$. For $x \in X$ and $f \in C$ let $f_{x} \in B$ be defined by $f_{x}(g)=f\left(\sigma_{g}(x)\right)$. Let $A=A(X) \subseteq B$ be the smallest uniformly closed algebra containing $\left\{f_{x}: x \in X, f \in C\right\}$. We slightly abuse notation to use the same symbol $f_{x}$ for the corresponding function defined on $G_{E}$. If $x \in X$, and $f \in C$ define $\overline{f_{x}}: E \rightarrow \mathbf{R}$ by $\overline{f_{x}}(\eta)=f(\eta(x))$. Observe that each $\overline{f_{x}}$ is in $C_{\mathbf{R}}(E)$, the space of continuous real valued functions on $E$. Clearly $\overline{f_{x}}$ is the continuous extension of $f_{x}$ from $G_{E}$ to $E$. It follows that every $\phi \in A$ has a (unique) continuous extension $\bar{\phi}: E \rightarrow \mathbf{R}$.

Proposition 1. $\{\bar{\phi}: \phi \in A\}=C_{\mathbf{R}}(E)$. In particular, $A=B$ if and only if the embedding $g \rightarrow \sigma_{g}$ is a realization of the Stone-Čech compactification $\beta$ of $G$.

Proof. It is clear that $\{\bar{\phi}: \phi \in A\} \subseteq C_{\mathbf{R}}(E)$ is a uniformly closed subalgebra of $C_{\mathbf{R}}(E)$. If $\xi$ and $\eta$ are distinct elements of $E$ then there is some $x \in X$ with

Received by the editors August 29, 1996.

1991 Mathematics Subject Classification. Primary 58F08, 58F03, 54H20.

Key words and phrases. Enveloping semigroup, subshift, topological action. 
$\xi(x) \neq \eta(x)$. Choose $f \in C$ so that $f(\xi(x)) \neq f(\eta(x))$, and thus $\overline{f_{x}}(\xi) \neq \overline{f_{x}}(\eta)$. This shows that $\{\bar{\phi}: \phi \in A\}$ separates points of $E$ and so, by the Stone-Weierstrass theorem, $\{\bar{\phi}: \phi \in A\}=C_{\mathbf{R}}(E)$.

As is well-known, a realization of the Stone-Cech compactification of the discrete space $G$ consists of a compact space $E$ and an injective mapping $g \rightarrow \sigma_{g}$ of $G$ onto a dense subspace $G_{E}$ of $E$ such that:

(1) The set $G_{E}$ is discrete as a subspace of $E$.

(2) Every bounded function on $G_{E}$ extends to a continuous function on $E$.

In fact (1) follows from (2) and (2) is simply the assertion that $A=B$. We have assumed $g \rightarrow \sigma_{g}$ is injective, and $G_{E}$ is dense by the definition of $E$. This shows that $E$ is the Stone-Cech compactification when $A=B$. The converse assertion is an immediate consequence of (2).

Remark. A similar result holds if $G$ has a completely regular topology and the action $\sigma$ of $G$ on $X$ is jointly continuous.

Proposition 2. Let I be any non-void index set. Let $X^{I}$ be the product space with the product topology and let $\sigma^{I}$ be the product action on $X^{I}$, defined by $\sigma_{g}^{I}(\bar{x})_{i}=$ $\sigma_{g}\left(\bar{x}_{i}\right)$ for $\bar{x} \in X^{I}$ and $i \in I$. Then $A\left(X^{I}\right)=A(X)$.

Proof. Clearly $A(X) \subseteq A\left(X^{I}\right)$, so we establish the reverse inclusion. Let $\pi_{i}(\bar{x})=\bar{x}_{i}$ for $\bar{x} \in X^{I}$ and $i \in I$. Clearly $\left\{f \circ \pi_{i}: f \in C, i \in I\right\}$ separates points of $X^{I}$. Given $f \in C_{\mathbf{R}}\left(X^{I}\right)$ and $\epsilon>0$, it follows from the Stone-Weierstrass theorem that there exist a positive integer $k$, functions $f^{1}, \ldots, f^{k} \in C$, indices $i_{1}, \ldots, i_{k} \in I$, and a real polynomial $p$ of $k$ variables such that $\left|f(\bar{x})-p\left(f^{1}\left(\pi_{i_{1}}(\bar{x})\right), \ldots, f^{k}\left(\pi_{i_{k}}(\bar{x})\right)\right)\right|<\epsilon$ for all $\bar{x} \in X^{I}$. Examination of the $\sigma^{I}$ action shows that

$$
\left|f_{\bar{x}}(g)-p\left(f_{x_{1}}^{1}(g), \ldots, f^{k}{ }_{x_{k}}(g)\right)\right|<\epsilon .
$$

Since $A(X)$ consists of the uniform closure of just such polynomial expressions, this inequality shows $f \in A(X)$.

We will apply the above results to actions on a totally disconnected space. We begin with a general definition of a shift space on a finite set.

Definition. Let $\alpha$ be a finite set with the discrete topology. Let $X=\alpha^{G}$ be the product space with the product topology. Let $\sigma$ be defined by $\left(\sigma_{g}(x)\right)_{h}=x_{h g}$. We call $(X, G, \sigma)$ the $G$-shift on $\alpha$.

Remark. The mapping $\sigma$ defined above is an action, and $g \neq h \Longrightarrow \sigma_{g} \neq \sigma_{h}$.

Proof. First observe $\left(\sigma_{g h}(x)\right)_{t}=x_{t g h}=\left(\sigma_{h}(x)\right)_{t g}=\left(\sigma_{g}\left(\sigma_{h}(x)\right)\right)_{t} \forall g, h, t \in G$ and $x \in X$. This shows $\sigma_{g h}=\sigma_{g} \circ \sigma_{h}$. Because $X$ has the product topology, evaluation $x \rightarrow x_{t g}$ is continuous for fixed $t$ and $g$. But continuity of $\sigma_{g}: X \rightarrow X$ is equivalent to continuity of $x \rightarrow\left(\sigma_{g}(x)\right)_{t}=x_{t g}$ at every $x \in X$ and for every $t \in G$. This shows each $\sigma_{g}$ is continuous. Finally, we have assumed the existence of an element $g_{0} \in G$ such that $g \rightarrow g_{0} g$ is injective. Given distinct $g$ and $h$ in $G$ let $x \in X$ be such that $x_{g_{0} g} \neq x_{g_{0} h}$. Then $\sigma_{g}(x) \neq \sigma_{h}(x)$ since $\left(\sigma_{g}(x)\right)_{g_{0}} \neq\left(\sigma_{h}(x)\right)_{g_{0}}$.

Proposition 3. Let $(X, G, \sigma)$ be a $G$-shift on some finite set $\alpha$ of cardinality greater than 1. Then $A_{X}=B$, and so $E_{X}$ is the Stone-Čech compactification of $G$. 
Proof. Clearly it is sufficient to show that for any subset $U \subseteq G$, the characteristic function $\chi=\chi_{U}$ of $U$ is in $A$. We can map $\alpha$ bijectively to $\{0,1, \ldots, k-1\}$ for some $k>1$ and for notational simplicity we assume that $\alpha$ in fact equals $\{0,1, \ldots, k-1\} \subset \mathbf{R}$. Choose $g_{0} \in G$ so $g \rightarrow g_{0} g$ is injective, and let $V=g_{0} U$. Define $\hat{x} \in X$ as the characteristic function of $V$. Define $f: X \rightarrow \mathbf{R}$ by $f(x)=x_{g_{0}}$. Observe $f_{\hat{x}}(g)=f\left(\sigma_{g}(\hat{x})\right)=\left(\sigma_{g}(\hat{x})\right)_{g_{0}}=\hat{x}_{g_{0} g}=\chi(g)$.

Definition. If $(X, G, \sigma)$ and $(Y, G, \tau)$ are flows then a continuous mapping $\Pi$ : $X \rightarrow Y$ is called a flow mapping if $\tau_{g}(\Pi(x))=\Pi\left(\sigma_{g}(x)\right)$ for all $x \in X$ and $g \in G$. If also $\Pi$ is surjective then we call $\Pi$ a factor mapping of $X$ onto $Y$ and we call $Y$ a factor of $X$.

Theorem 1. Let $(X, G, \sigma)$ be an action of the discrete semigroup $G$ on the compact totally disconnected Hausdorff space $X$, satisfying $g \neq h \Longrightarrow \sigma_{g} \neq \sigma_{h}$. Assume that there is an element $g_{0} \in G$ such that $g \rightarrow g_{0} g$ is injective. Let $A(X), B$ and $E_{X}$ be as above. Let $(Y, G, \tau)$ be the $G$-shift on the two symbol set $\alpha=\{0,1\}$. Assume that there is at least one $\hat{y} \in Y$ such that $\left\{\tau_{g}(\hat{y}): g \in G\right\}$ is dense in $Y$ (i.e., $(Y, G, \tau)$ is transitive). Then the following four statements are equivalent:

(1) For some finite non-void index set $I$, the shift action $(Y, G, \tau)$ is a factor of the product action $\left(X^{I}, G, \sigma^{I}\right)$.

(2) For some non-void index set $I$, the shift action $(Y, G, \tau)$ is a factor of the product action $\left(X^{I}, G, \sigma^{I}\right)$.

(3) $A=B$.

(4) $E_{X}$, together with the mapping $g \rightarrow \sigma_{g}$, is a realization of the Stone- $\breve{C} e c h$ compactification of $G$.

Proof. Clearly (1) $\Longrightarrow(2)$ and we have seen from Proposition 1 that $(3) \Longleftrightarrow$ (4). Also $(2) \Longrightarrow B=A(Y)$ by Proposition 3 , but $A(Y) \subseteq A\left(X^{I}\right)=A(X)$ by Proposition 2, and $A(X) \subseteq B$. So $(2) \Longrightarrow(3)$.

We complete the proof by showing $(3) \Longrightarrow(1)$.

Assume (3) holds. Select $\hat{y}$ such that $\left\{\sigma_{g}(\hat{y}): g \in G\right\}$ is dense in $Y$. Since $\hat{y}$ has domain $G$ and values in $\{0,1\}$ it is an element of $B$. From (3) and by the Stone-Weierstrass theorem, there exist a positive integer $k$, functions $f^{1}, \ldots, f^{k}$ in $C$, points $\hat{x}_{1}, \ldots, \hat{x}_{k}$ in $X$, and a polynomial $p$ in $k$ variables so that

$$
\left|p\left(f_{\hat{x}_{1}}^{1}(g), \ldots, f_{\hat{x}_{k}}^{k}(g)\right)-\hat{y}_{g}\right|<\frac{1}{4}
$$

for all $g \in G$. Since $X$ is totally disconnected, any two points of $X$ can be distinguished by some continuous function that assumes only two values. Again using the Stone-Weierstrass theorem, we may rechoose $k, p$, and the $f^{i}$, and renumber the $x_{i}$ so that, in addition to the above inequality, the range $T$ of $p\left(f_{x_{1}}^{1}(g), \ldots, f_{x_{k}}^{k}(g)\right)$ is a finite set. Let $\gamma \in\left(\frac{1}{4}, \frac{3}{4}\right) \backslash T$ and let $\chi$ be the characteristic function of $[\gamma, \infty)$. Define $\Pi: X^{k} \rightarrow Y$ by $\Pi\left(x_{1}, \ldots, x_{k}\right)(g)=\chi\left(p\left(f_{x_{1}}^{1}(g), \ldots, f_{x_{k}}^{k}(g)\right)\right)$. The map $\Pi$ is continuous on $X$ since $\chi$ is continuous on $T$. Calculate

$$
\begin{aligned}
& \tau_{h}\left(\Pi\left(x_{1}, \ldots, x_{k}\right)\right)(g)=\Pi\left(x_{1}, \ldots, x_{k}\right)(g h)=\chi\left(p\left(f_{x_{1}}^{1}(g h), \ldots, f_{x_{k}}^{k}(g h)\right)\right) \\
& \quad=\chi\left(p\left(f^{1}\left(\sigma_{g h}\left(x_{1}\right), \ldots, f^{k}\left(\sigma_{g h}\left(x_{k}\right)\right)\right)\right)\right) \\
& \quad=\chi\left(p\left(f^{1}\left(\sigma_{g}\left(\sigma_{h}\left(x_{1}\right)\right)\right), \ldots, f^{k}\left(\sigma_{g}\left(\sigma_{h}\left(x_{k}\right)\right)\right)\right)\right)=\Pi\left(\sigma_{h}^{I}\left(x_{1}, \ldots, x_{k}\right)\right)(g),
\end{aligned}
$$

so $\Pi$ is a flow mapping. Finally, $\Pi: X^{I} \rightarrow Y$ is surjective since $\Pi\left(\hat{x}_{1}, \ldots, \hat{x}_{k}\right)=$ $\hat{y}$. 
The hypothesis in Theorem 1 requiring $Y$ to be transitive is impractical as it may not be easily verifiable. In contrast, the transitivity of $Y$ follows from the following modest condition on $G$, when the cardinality of $G$ is infinite, which is easy to check:

Definition. $G$ is said to satisfy the Separation Condition if the cardinality $|G|$ of $G$ is infinite and if for all $h, k \in G,|\{g \in G: h g=k\}|<|G|$.

Theorem 2. If $G$ satisfies the Separation Condition, then the shift space $(Y, G, \tau)$ is transitive.

We first need the lemma:

Lemma. Let $G$ be an infinite semigroup and let $G_{0}$ and $G_{1}$ be a partition of $G$. Let $y^{c}$ be the characteristic function of $G_{1}$. Then $y^{c}$ has dense $\tau$-orbit if and only if whenever $F_{0}$ and $F_{1}$ are disjoint finite subsets of $G$, there is an element $g \in G$ such that $F_{0} g \subseteq G_{0}$ and $F_{1} g \subseteq G_{1}$.

Proof (of Lemma). "if": Pick $y \in Y$ and let $F$ be a finite subset of $G$. Let $F_{i}=$ $\left\{g \in G: y_{g}=i\right\}, i=0,1$. Choose $g \in G$ such that $F_{i} g \subseteq G_{i}, i=0,1$. Pick $h_{i} \in F_{i}$. Then $\left(\sigma_{g} y^{c}\right)_{h_{i}}=y_{h_{i} g}^{c}=i=y_{h_{i}}$. This shows that given $y$ and $F$, there is a $g$ such that $\sigma_{g} y^{c}$ agrees with $y$ on $F$. That is, the orbit of $y^{c}$ is dense in $Y$.

The converse is proven similarly.

Proof (of Theorem 2). We construct sets $G_{i}^{\zeta}, i=0,1$, inductively, using transfinite induction to account for uncountable $G$. It will be clear from the construction that $G_{i}^{\eta} \subseteq G_{i}^{\nu}$ if $\eta<\nu$.

For any ordinal $\eta$, we let $\bar{\eta}$ be the set of strict predecessors of $\eta$. Now let $\xi$ be the least ordinal such that $|\bar{\xi}|=|G|$. Let $\mathcal{F}$ consist of all ordered pairs of disjoint finite subsets of $G$. Let $\zeta \rightarrow\left(F_{0}^{\zeta}, F_{1}^{\zeta}\right)$ be a bijective mapping of $\bar{\xi}$ onto $\mathcal{F}$.

Set $G_{0}^{0}=G_{1}^{0}=\emptyset$.

Suppose that for some ordinal $\zeta$ with $|\bar{\zeta}|<|G|$ the sets $G_{0}^{\zeta}$ and $G_{1}^{\zeta}$ have been defined such that $G_{0}^{\zeta} \cap G_{1}^{\zeta}=\emptyset$ and $\left|G_{i}^{\zeta}\right|<|G|, i=0,1$. Choose $g_{\zeta} \in G$ such that the four sets $G_{0}^{\zeta}, G_{1}^{\zeta}, F_{0}^{\zeta} g_{\zeta}, F_{1}^{\zeta} g_{\zeta}$ are pairwise disjoint. Such a $g_{\zeta}$ exists since $\left|G_{0}^{\zeta} \cup G_{1}^{\zeta} \cup F_{0}^{\zeta} \cup F_{1}^{\zeta}\right|<|G|$ and since $G$ satisfies the Separation Condition. Set $G_{i}^{\zeta+1}=G_{i}^{\zeta} \cup F_{i}^{\zeta} g_{\zeta}$. Then $G_{0}^{\zeta+1}$ and $G_{1}^{\zeta+1}$ have been defined, are disjoint, and have cardinality strictly less than that of $G$.

If $\zeta$ is a limit ordinal with $|\bar{\zeta}|<|G|$ and if $G_{i}^{\eta}, i=0,1$, for all $\eta<\zeta$, then set $H_{i}^{\zeta}=\cup_{\eta<\zeta} G_{i}^{\eta}$ and choose $g_{\zeta}$ so that $H_{0}^{\zeta}, H_{1}^{\zeta}, F_{0}^{\zeta} g_{\zeta}, F_{1}^{\zeta} g_{\zeta}$ are pairwise disjoint. Set $G_{i}^{\zeta}=H_{i}^{\zeta} \cup F_{i}^{\zeta}$.

Finally, set $G_{0}=\cup_{\eta<\zeta} G_{0}^{\eta}$ and $G_{1}=G \backslash G_{0}$. Let $y^{c}$ be the characteristic function of $G_{1}$. It is now easy to check that if $\left(F_{0}^{\zeta}, F_{1}^{\zeta}\right) \in \mathcal{F}$, then $g_{\zeta}$ satisfies the condition of the lemma.

We now consider the case $G=(\mathbf{Z},+)$, the integers under addition. We let $\sigma=\sigma_{1}$ and then $\sigma^{n}=\sigma_{n}$.

It is well-known that a mixing subshift of finite type has a Cartesian product with the full 2-shift as a factor (see [2]) and so the enveloping semigroup $E$ is the Stone-Čech compactification $\beta$ of $\mathbf{Z}$. The first theorem in [6] implies that no flow "constructed from minimal flows" can have $E=\beta$. Let us be more precise: A bounded function $F: \mathbf{Z} \rightarrow \mathbf{R}$ is called minimal if for every finite $F \subset \mathbf{Z}$ and every 
$\epsilon>0$ the set $\{n \in \mathbf{Z}:|F(n+i)-F(i)|<\epsilon \forall i \in F\}$ is syndetic in $\mathbf{Z}$. Let $\mathcal{U}$ be the smallest uniformly closed algebra containing every minimal function. It is shown in [6] (Theorem 1 ) that $\mathcal{U}$ is properly contained in $B$, the algebra of bounded real valued functions defined on $\mathbf{Z}$. A $\mathbf{Z}$-flow $X$ is minimal if there are no closed invariant non-void proper subsets. This condition is equivalent to: For every $x \in X$ and open set $U$ containing $x$, the set $\left\{n \in \mathbf{Z}: \sigma^{n}(x) \in U\right\}$ is syndetic. From this it is easy to see that for a minimal flow $X$ every $f_{x}$ is a minimal function. It follows that every minimal flow $X$ has $A \neq B$. Moreover, if we take Cartesian products, subflows, or factors of flows satisfying $A \subseteq \mathcal{U}$ then the resulting flow will also have $A \subseteq \mathcal{U}$ and so $E \neq \beta$. We know, both from specific construction (see for instance [7]) and from the Jewitt-Krieger theorem, that there are many minimal subshifts of positive entropy.

Before giving the next example it is convenient to introduce some terminology. Let $(X, d)$ be a compact metric space and let $\sigma$ be a homeomorphism of $X$. Say that a point $x \in X$ has an arithmetically clustered orbit if for every $\epsilon>0$ there is an arithmetic subset $S=a+L \mathbf{Z} \subseteq \mathbf{Z}(a, L \in \mathbf{Z})$ such that if $n, m \in S$ then $d\left(\sigma^{n} x, \sigma^{m} x\right)<\epsilon$. We say $(X, \sigma)$ clusters arithmetically, or is of class $\mathcal{A}$, if every point of $X$ has an arithmetically clustered orbit. The property of arithmetically clustered is unchanged if $d$ is replaced by some other topologically equivalent (hence uniformly equivalent, since $X$ is compact) metric.

Proposition 4. (1) If $(X, \sigma)$ and $(Y, \tau)$ are in $\mathcal{A}$ then $(X \times Y, \sigma \times \tau)$ is in $\mathcal{A}$.

(2) If $(X, \sigma)$ is in $\mathcal{S}$ and if $(Y, \tau)$ is a factor of $(X, \sigma)$ then $(Y, \tau)$ is in $\mathcal{S}$.

(3) The 2-shift does not cluster arithmetically.

Proof. (1) Let $(x, y) \in X \times Y$ and let $\epsilon>0$. We use the same symbol $d$ to denote the metric on each space. It suffices to find an arithmetic set $S$ such that $n, m \in S$ implies $d\left(\sigma^{n} x, \sigma^{m} x\right)<\epsilon$ and $d\left(\tau^{n} x, \tau^{m} x\right)<\epsilon$. Let $S_{x}=a+L \mathbf{Z}$ be the appropriate arithmetic set for $x$ and $\epsilon$. Choose $\delta>0$ so that if $y_{1}, y_{2}$ in $Y$ and $d\left(y_{1}, y_{2}\right)<\delta$ then $d\left(\tau^{k} y_{1}, \tau^{k} y_{2}\right)<\epsilon$ for $0 \leq k \leq L-1$. Now choose $S_{Y}=b+M \mathbf{Z}$ so that $m, n \in S_{Y}$ implies $d\left(\tau^{m} y, \tau^{n} y\right)<\delta$. Choose $u \in \mathbf{Z}$ and $0 \leq k \leq L-1$ so that $a+u L=b+k$. Let $S=a+u L+L M \mathbf{Z}$. Observe that $S \subseteq S_{x}$ so $n, m \in S$ implies $d\left(\sigma^{n} x, \sigma^{m} x\right)<\epsilon$. Write $S$ as $b+k+L M \mathbf{Z}$. If $n, m \in S$ then $n-k, m-k \in S_{Y}$ so $d\left(\tau^{n-k} y, \tau^{m-k} y\right)<\delta$ yielding $d\left(\tau^{n} y, \tau^{m} y\right)<\epsilon$.

(2) This is a direct consequence of uniform continuity.

(3) The point $0,1,1,0,0,0,1,1,1,1, \ldots$ does not have an arithmetically clustered orbit.

Remark. Theorem 1 implies that no zero dimensional $\mathcal{A}$ flow can have its enveloping semigroup equal to $\beta$. The dimensional assumption is superfluous. Suppose $(X, \sigma)$ clusters arithmetically and suppose the function $f$ is in the corresponding algebra $A(X)$. It is easy to show that for every $\epsilon>0$ there is an arithmetic set $S$ such that if $n, m \in S$ then $|f(n)-f(m)|<\epsilon$. It follows that $A \neq B$ and so $E \neq \beta$.

The next example, a mild variant of one provided by B. Weiss at the 1995 CBMS conference in Bakersfield, is a non-minimal subshift (of the 2-shift) of positive entropy. It belongs to the class $\mathcal{A}$ and so $E \neq \beta$.

Let $m=m_{1}, m_{2}, \cdots \in\{0,1,2,3, \ldots, 9\}^{\mathbf{N}}=M$. With each $m \in M$ we associate $I_{m} \subseteq \mathbf{Z}$ defined by $i \in I_{m}$ if and only if there exist $n \in \mathbf{N}$ and $k \in \mathbf{Z}$ such that 
$\left|i-k 10^{n}-\sum_{j=1}^{n} m_{j} 10^{j-1}\right|<n$. For example, if $m=3,2,5 \ldots$ then, for any $k, 3+10 k \in I_{m},\{22,23,24\}+100 k \subset I_{m},[521,525]+1000 k \subset I_{m} \ldots$ We let $x \in X \subset\{0,1\}^{\mathbf{Z}}$ if there is some $m \in M$ such that $x_{i}=0$ for each $i \in I_{m}$. We will say such an $x$ and $m$ are paired. In general neither $x$ nor $m$ determines the other. We let $\sigma$ be the shift: $\sigma(x)_{i}=x_{i+1}$.

Proposition 5. $X$ is a closed shift invariant set and the flow $(X, \sigma)$ has positive topological entropy.

Proof. If $x \in X$ and $m \in M$ are paired in the above manner then $m-1$ and $m+1$ are paired with $\sigma(x)$ and $\sigma^{-1}(x)$ respectively, where $m+1$ is obtained by changing the leading string of nines (if there is one) to zeroes and then increasing the first non-nine digit (if there is one) by 1 , and $m-1$ is defined analogously so that $(m+1)-1=m$.

Now suppose $x^{(\nu)}$ is a sequence of points in $X$ converging to a point $x$ in $\{0,1\}^{\mathbf{Z}}$. We will show $x \in X$. Let $m^{\nu} \in M$ be paired with $x^{\nu}$. Since $M$ is compact, we can assume (passing to a subsequence if necessary) that $m^{\nu}$ converges to some $m \in M$. It is easily seen that $x$ and $m$ are paired. This shows $X$ is closed.

Next let $X_{1}$ be the set of $x$ paired with $\mathbf{1}=1,1,1, \ldots$ If we look at the entries in the interval $\left[1,10^{k}\right]$ we see that ones are specified at $I_{\mathbf{1}} \cap\left[1,10^{k}\right]$ and any pattern of zeroes and ones may appear at the remaining entries. The set $I$ consists of $\{1,11,21, \ldots\} \cup\{10,12,110,112, \ldots\} \cup\{109,113,1109,1113, \ldots\} \cup \ldots$ Thus

$$
\begin{aligned}
{\left[\operatorname{card}\left(I \cap\left[1,10^{k}\right]\right)\right.} & <10^{k}\left(\frac{1}{10}+\frac{2}{10^{2}}+\frac{2}{10^{3}}+\ldots\right) \\
& =10^{k}\left(\frac{1}{10}+\frac{2}{10^{2}} \frac{1}{1-\frac{1}{10}}\right)=\frac{11}{90} \times 10^{k} .
\end{aligned}
$$

A finite block $x_{1}, \ldots, x_{n}$ in $\{0,1\}^{n}$ is called an admissible $n$-block if there is some two-side continuation $\ldots, x_{1}, \ldots, x_{n} \ldots$ to an element of $X$. We have just shown that for $n=2^{k}$ the number of admissible $n$-blocks is at least $2^{\frac{79}{90} n}$. This, as is well-known, implies that $\sigma$ has positive entropy.

It is obvious from the construction that $(X, \sigma)$ belongs to $\mathcal{A}$.

\section{ACKNOWLEDGMENTS}

As noted, this last example is due to B. Weiss. We thank H. Furstenberg for the reference [6], and for pointing out the relevance of their Theorem 1. Conversations with M. Boyle, J. Auslander and CSUB student Andrew Sean Watson were helpful.

\section{REFERENCES}

1. J. Auslander, Minimal Flows and their Extensions, North-Holland, 1988. MR 89m:54050

2. M. Boyle, Lower Entropy Factors on Topological Dynamics, Erg. Th. and Dyn. Syst. 4 (1984), 541-557. MR 85m:54014

3. R. Ellis, A Semigroup Associated with a Transformation Group, Trans. Amer. Math. Soc. 94 (1960), 272-281. MR 23:A961

4. R. Ellis, Lectures on Topological Dynamics, W. A. Benjamin, 1969. MR 42:2463

5. H. Furstenberg, Recurrence in Ergodic Theory and Combinatorial Number Theory, Princeton Univ. Press, Princeton, NJ, 1981. MR 82j:28010 
6. S. Glasner and B. Weiss, Interpolation Sets For Subalgebras of $l^{\infty}(\mathbf{Z})$, Israel Journal of Mathematics 4 (1983), 345-360. MR 85f:46098

7. F. Hahn and Y. Katznelson, On the Entropy of Uniquely Ergodic Transformations, Trans. Amer. Math. Soc. 126 (1967), 335-360. MR 34:7772

Department of Mathematics, University of Maryland at College Park, College PARK, MARYLAND 20742

E-mail address: krb@hroswitha.umd.edu

Department of Mathematics, California State University at Bakersfield, BakersFIELD, CALIFornia 93311

E-mail address: dgove@ultrix6.cs.csubak.edu

Department of Mathematics, California State University at Bakersfield, BakersFIELD, CALIFORNIA 93311

E-mail address: khaddad@ultrix6.cs.csubak.edu 